\title{
QUELLEN
}

Reglement für die Eidg. Maturitätsprüfungen vom 2o. Januar 1925 (Stand Dezember 1954). - Revision der Eidg. Verordnung über die Anerkennung der Maturitätsausweise. Bericht der Eidg. Expertenkommission. - Artikel Vaterlaus in NZZ 28. April 1959 und 3. Juli 1964. H. Bernhard: Der Geographieunterricht in der Schweiz in 'Geographische Rundschaus, März 1963. - H. Bernhard: Die Geographie in den Stundentafeln verschiedener Länder Europas in ‘Geographica Helvetica) 1965.

\section{EINE INTERNATIONALE ARBEITSGRUPPE FÜR DIE GEOGRAPHISCHE TERMINOLOGIE DER AGRARLANDSCHAF'T}

\author{
WERNER GALLUSSER und HAROLD HAEFNER
}

Unter Leitung von Prof. Dr. H. Uhlig, Geographisches Institut der Universität Gießen, hat sich eine internationale Arbeitsgruppe gebildet, der alle agrargeographisch interessierten deutschen Geographen und zahlreiche Vertreter aus 25 weiteren Ländern angehören. Das Ziel dieser internationalen Gemeinschaftsarbeit besteht in der Erstellung eines Glossars, das alle agrargeographischen Fachausdrücke definiert [nach a) der erstmals verwendeten Fassung oder einem Standardwerk und b) dem neuesten Stand der Forschung], alternative Begriffe (regionale Varianten) und den entsprechenden fremdsprachigen Terminus nennt, oder aufzeigt, was unter einem bestimmten Begriff in einem Sprachgebiet verstanden wird.

Wer um die begriffliche Verwirrung weiß und die Gefahr des Aneinander-vorbei-Redens kennt (denken wir nur an den Ausdruck (Transhumanz)), der wird es freudig begrüßen, daß ein derartiges Werk an die Hand genommen wird.

Um eine Vorstellung über Aufbau und Umfang des geplanten Glossars geben zu können, fügen wir die vorgeschlagene provisorische Aufgliederung der Begriffe nach Sachgruppen in einem Dezimalsystem an:

o Allgemeine Grundbegriffe der Kulturforschung, der Agrargeographie und der Agrargeschichte.

o.1 Kulturwissenschaftliche Grundbegriffe (Wirtschafts- und Kulturstufen usw.).

o.2 Agrargeographische Grundbegriffe (Landwirtschaft, Agrargeographie).

o.3 Agrargeschichtliche Grundbegriffe (Villikation, Zehnt).

1 Agrargeographische Landschaftstypen, -zonen, -grenzen usw.

1.o Agrageographisch relevante (natürliche oder anthropogen gewandelte) Landschaftstypen und typische Standorte (Marsch, Puszta).

1.1 Kulturgeographisch-genetische Landschaftstypen (Altsiedelland, Rodungsgebiet).

1.2 Agrargeographische Zonen (kont. Sommerweizenzone, Alpine Stufe).

1.3 Agrargeographisehe Grenzen (Trockengrenze, Höhengrenze der Dauersiedlung).

2 Siedlungen und Siedlungsentwicklung.

2.o Siedlungsformen (Weiler, Angerdorf).

2.1 Funktionelle Siedlungstypen (Sommerdorf, Alpsiedlung, Weingut).

2.2 Siedlungsgenetische Begriffe, Landerschließung und Landgewinnung (Aussiedlerhof, Landnahme, Wüstung).

2.3 Hausformen, -typen, -teile (Vierseithof, Gotthardhaus).

3 Flur- und Nutzflächengefüge.

3.o Gemarkungsorganisation, Besitz- und Nutzungsformen bzw. -areale (Gemarkung, Allmende).

3.1 Flurformen und Flurteile (Zelge, Blockflur).

3.2 Besitzmaße und -einheiten und Ackermaße (Hufe, Tagewerk).

3.3 Intensitäts-, Anbau-, Flurzonen (Wildland, Thünensche Ringe).

3.4 Einhegungen, Feld- und Flurgrenzen (Windschutzstreifen, Etter).

4 Landnutzung.

4.o Landwirtschaftliche Betriebsformen (Gemischtbetriebe, Grünlandwirtschaft).

4.1 Wechselwirtschaftsformen (shifting-cultivation, Egartenwirtschaft).

4.2 Viehwirtschaft (Viehzucht, Transhumanz, Nomadismus).

4.3 Ackerbau (Dreifelderwirtschaft, Regenfeldbau).

4.4 Künstliche Bewässerung (Bewässerungswiesen, Schöpfrad, bisse) 
4.5 Bäuerliche Baumkulturen, cultura mista, Weinbau und andere Sonderkulturen; Marktgartenbau (Obstwiese, Olivenhain).

4.6 Plantagenwirtschaft (Plantage, Pflanzung, Monokultur).

4.7 Bäuerliche Holz-, Wald- und Heidenutzung, Bienenzucht, Fischzucht und andere Ergänzungswirtschaften (Schneiteln, Waldweide).

5 (Agrar-) Sozialgeographische Begriffe.

5.o Soziale Typen des ländlichen Menschen (Farmer, Kätner).

5.1 Agrarsoziale Organisationsformen und -gruppen (Genossenschaft, Grundherrschaft).

5.2 Sozialstruktur und Besitzformen (Großgrundbesitz, Kollektivwirtschaft).

5.3 Besondere agrarsoziale Phänomene (Sozialbrache, Entmischung).

5.4 Erbformen (Realteilung, Majorat).

6 Wirtschaftliche, kultur- und arbeitstechnische sowie agrarstatistische Begriffe.

6.o Betriebstechnische Begriffe (Fruchtfolge, Brache).

6.1 Kulturtechnische Begriffe (Flurbereinigung, Melioration).

6.2 Agrarstatistische Begriffe (Großvieheinheiten, Nutzfläche).

Im Hinblick auf die internationale Bedeutung, die einer derartigen terminologischen Läuterung zukommt, sollte auch die Schweizer Geographie ihre Mithilfe nicht versagen, nicht zuletzt auch wegen der möglichen Vermittlerfunktion zwischen der deutschen und französischen oder italienischen Fachsprache.

Die beiden Schreibenden haben es unternommen, die Mitarbeit der schweizerischen Geographen anzuregen und für eine erste Organisation der Arbeit zu sorgen. Als Anfang möchten wir nun die interessierten inländischen Fachkollegen ermuntern, sich für die Gemeinschaftsarbeit zur Verfügung zu stellen unter Bezeichnung jener Begriffsgruppen des obigen Systems, bei denen man mitzuwirken wünscht. Wenn in Einzelfällen keine Mitarbeit geleistet werden kann, sind wir auch für kurze Mitteilungen dankbar, in denen auf wichtige Schweizer Begriffe hingewiesen wird, die im Glossar enthalten sein sollten. Ferner dienen auch Angaben über bestimmte Fachleute, die für einzelne Sachfragen zu Rate gezogen werden sollten.

Bei den Zusendungen wären demnach folgende drei Punkte zu beachten:

1. Mitwirkung in welchen Begriffsgruppen?

2. Welche Begriffe sollten Ihrer Meinung nach unbedingt geklärt werden?

3. Welche Fachleute schlagen Sie für einzelne Begriffsgruppen vor?

Auf Grund der einlaufenden Meldungen werden wir das weitere Vorgehen und die Verteilung der vorgedruckten Arbeitsblätter koordinieren. Ihre Mitteilungen bitten wir zu richten an: Dr. W. Gallusser, Geographisches Institut der Universität, 4000 Basel, Klingelbergstraße 16.

\section{GESELLSCHAFTSTÄTIGKEIT - ACTIVITÉ DES SOCIÉTÉS}

Vorträge im Wintersemester 1965/66. Basel. 3o. Oktober: Exkursion, Lörrach - unbekannte Nachbarstadt. 5. November: Dr. C. Baer, Basel: Führung durch die Ausstellung \&Südamerikanische Indianer ; 19. November: Dr. E. Knuth, Kopenhagen: Früheste Einwanderungen nach Nordgrönland; 3. Dezember: O. Jenny, Oberdorf: Portugal auf Seitenwegen; 7. Januar: Dr. U. P. Büchi, Zürich: Das schweizerische Erdölproblem; 21. Januar: (Jahresversammlung), Dr. H. W. Muggli, Basel: Londons neue Satellitenstädte im Rahmen der englischen Landesplanung; 4. Februar: Dr. H. Schiffers, Köln: Die innersaharische Landschaft Borku und das TschadProblem; 18. Februar: Prof. Dr. J. Schubert, Leipzig: Reisen und Forschungen in der mongolischen Volksrepublik; 11. März: E. Schultheß, Zürich: China; 18. März: Dr. K. G. Siegler, Stuttgart: Kalabsha, der größte nubische Tempel und seine Versetzung. Bern. 19. Oktober: Prof. Dr. G. Grosjean, Bern: Bern-Beiträge zu einer Stadtgeographie; 29. Oktober: Prof. Dr. H. Bernhard, $Z$ ürich: Unterhaltendes und Belehrendes von einer Reise durch Nordamerika; 16. November: E. Knuth: Neue Aspekte zur Besiedlung von Grönland; 3o. November: K. Gähwyler: Südamerika — unbewältigter Kontinent; 14. Dezember: K. E. Larsson, Göteborg: Rewasan — ein Bergdorf auf den Fidschi-Inseln; 13. Januar: W. Dostal, Bern: Arabien; 25. Januar (Hauptversammlung) : M. Hohl, Bern: Verkehrsprobleme in der Agglomeration Bern; 1. Februar: Dr. H. Schiffers: Die innensaharische Landschaft; 15. Februar: H. Muggli: Londons neue Satellitenstädte; 1. März: E. Schultheß: China; 15. März: C. G. Siegler: Kalabsha. St. Gallen. 11. Oktober: M. de Vais- 